\title{
Belgeo
}

Revue belge de géographie

2| 2021

Dynamiques des campagnes et adaptations aux enjeux contemporains (Nord et Sud)

\section{Patrimoine rural et développement local dans les campagnes mecklembourgeoises (Allemagne) : quelles alternatives à la périphérisation ?}

Rural heritage and local development in the Mecklenburg countryside

(Germany): what alternatives to peripheralization?

Joséphine Lécuyer

\section{(2) OpenEdition}

Journals

Édition électronique

URL : https://journals.openedition.org/belgeo/49009

DOI : 10.4000/belgeo.49009

ISSN : 2294-9135

Éditeur :

National Committee of Geography of Belgium, Société Royale Belge de Géographie

Référence électronique

Joséphine Lécuyer, «Patrimoine rural et développement local dans les campagnes

mecklembourgeoises (Allemagne) : quelles alternatives à la périphérisation ? », Belgeo [En ligne], 2 |

2021, mis en ligne le 11 juin 2021, consulté le 14 juin 2021. URL : http://journals.openedition.org/

belgeo/49009; DOI : https://doi.org/10.4000/belgeo.49009

Ce document a été généré automatiquement le 14 juin 2021.

Belgeo est mis à disposition selon les termes de la licence Creative Commons Attribution 4.0 International. 


\title{
Patrimoine rural et développement local dans les campagnes mecklembourgeoises (Allemagne) : quelles alternatives à la périphérisation?
}

Rural heritage and local development in the Mecklenburg countryside (Germany): what alternatives to peripheralization?

\author{
Joséphine Lécuyer
}

\section{Introduction}

1 Le concept de périphérisation a été développé en Allemagne à la fin des années 2000, et il y est de plus en plus préféré au terme de décroissance (Roth, 2016) pour désigner les processus conjoints de pertes migratoires, de décrochage économique, de dépendance, ainsi que de stigmatisation (Kühn, Weck, 2013). Cette approche se distingue de l'analyse en termes de déclin (Schrumpfung) (Florentin et al., 2009) en ce qu'elle est fondée sur l'asymétrie des flux, et les relations de dépendance des régions périphériques (Bernt $e t$ al., 2009). De plus, l'analyse par la Schrumpfung (qui correspond au champ des shrinking cities) a été popularisée par des urbanistes, tandis que le processus de périphérisation a d'abord été étudié par des sociologues ruralistes (Keim, 2006), et surtout a été davantage mobilisé pour analyser la trajectoire des espaces ruraux. Les espaces touchés par la périphérisation ne sont donc pas simplement des espaces au faible potentiel économique, mais bien des espaces appréhendés comme un construit social, pris dans des systèmes de dépendance (Copus, Noguera, 2016).

2 Le Mecklembourg-Poméranie-occidentale ${ }^{1}$ est un Land rural du nord-est de l'Allemagne. Le centre du territoire, éloigné des grandes villes et centres économiques, 
est particulièrement touché par la périphérisation. La région de Demmin $^{2}$, petite ville et ancienne préfecture de district rural, est représentative de ces difficultés.

Figure 1. Évolution de la population du Mecklembourg-Poméranie (taux de croissance annuel moyen entre 1991 et 2011).

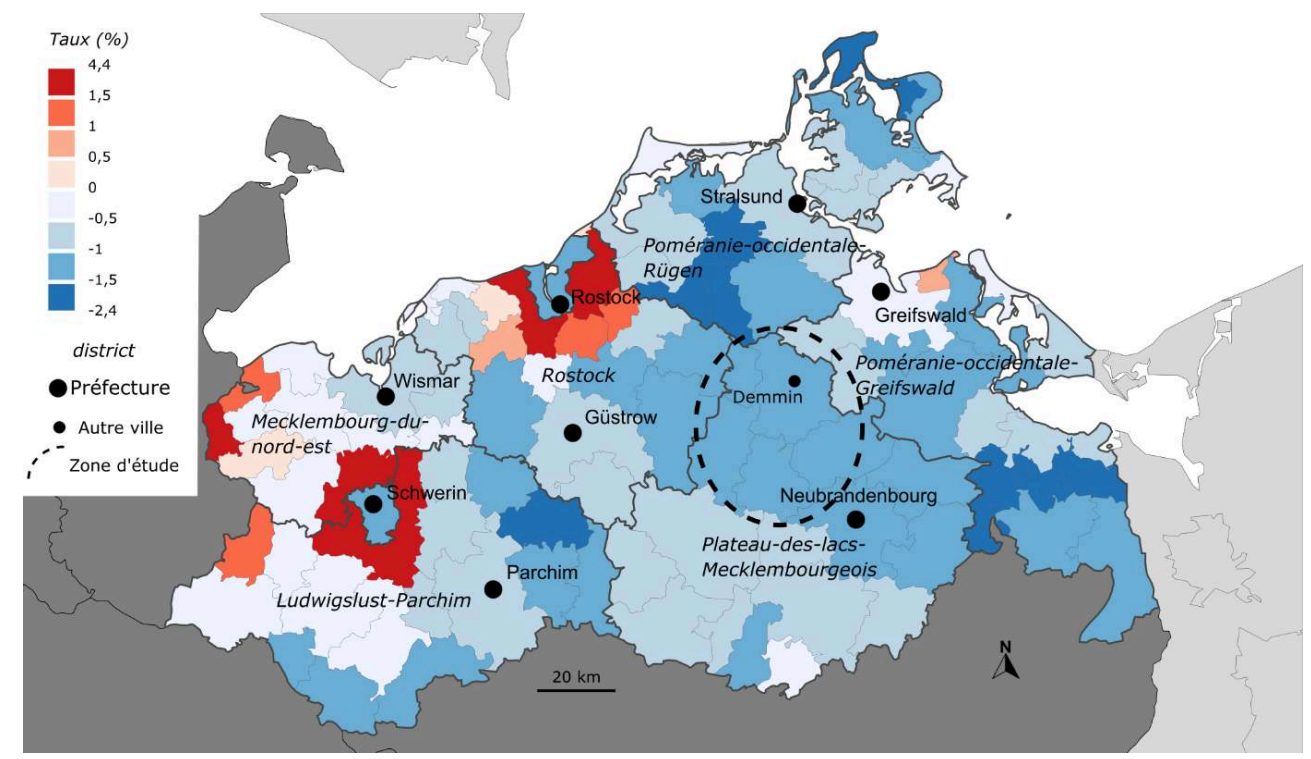

Source : Eurostat, 2011, réalisé avec Magrit

Dans cette région périphérisée, il existe a priori peu de perspectives de développement, en dehors de la valorisation du patrimoine, qui apparait alors souvent comme l'unique et meilleure ressource territoriale aux yeux des acteurs locaux. La mise en valeur de ce patrimoine naturel et bâti s'appuie sur le paysage caractéristique de la région (marais, lacs, châteaux et manoirs...).

4 Cependant, l'ensemble de cette valorisation semble s'effectuer principalement à destination d'autres personnes que les habitants (touristes citadins, nouveaux habitants potentiels), et sous injonction des échelons politiques supérieurs. Le développement local suppose une certaine autonomisation des acteurs locaux ; dans un contexte de périphérisation, leur marge de manœuvre semble relativement limitée. Cet article questionne donc le potentiel de développement local par la patrimonialisation dans une région périphérisée. Dans un contexte d'affaiblissement démographique et économique, dans quelle mesure les acteurs locaux d'un territoire dépendant et stigmatisé ont-ils les capacités d'impulser et de coordonner une action de construction patrimoniale? L'action collective de valorisation des ressources locales est-elle indépendante ou au contraire commandée depuis l'extérieur?

5 Cet article s'appuie sur un travail de terrain de quatre mois, mené dans le Mecklembourg-Poméranie en 2019 et 2020, au cours duquel ont été réalisés 36 entretiens avec des acteurs du développement local, ainsi qu'un travail d'observation lors d'une douzaine de réunions rassemblant des acteurs du développement local. Concernant la région de Demmin, le manager de la région LEADER ${ }^{3}$ Demminer Land a été le point d'entrée de l'enquête de terrain. Par la méthode des réseaux sociaux égocentrés, il a recommandé des contacts potentiels pour des entretiens dans sa région, qui ont eux-mêmes donné leurs propres contacts. Le panel des enquêtés comprend donc des porteurs de projets LEADER mais aussi des élus locaux et des techniciens de 
l'aménagement du territoire. L'objectif des entretiens semi-directifs n'était pas tant la collecte d'informations formelles (souvent disponibles en ligne) que le recueil de leur avis et impression sur leur région et ses perspectives de développement. Les enquêtés étaient donc relativement libres d'aborder les sujets qui leur paraissaient importants. De grands thèmes ont malgré tout été systématiquement abordés et faisaient l'objet de questions s'ils n'avaient pas été évoqués spontanément (évolution de la situation de la région au cours des dernières années, avenir de la région, perception des ressources et des faiblesses, partenaires de collaboration privilégiés/évités, meilleurs projets/projets ayant échoué, rapports avec le district et le Land). Une fois les premiers contacts établis, l'observation de réunions portant sur le développement local a été facilitée. En parallèle, les politiques publiques et documents d'aménagement concernant le développement des espaces ruraux à différents échelons (fédéral, Land, district, région LEADER, commune) ont été analysés.

\section{Le patrimoine, l'une des rares ressources locales dans un espace rural périphérisé}

6 Particulièrement touchée par des départs de populations importants et un affaiblissement économique généralisé, la région de Demmin a une image associée au vide et au déclin. Elle dispose cependant d'un patrimoine, en particulier naturel, important. Les dispositifs de certification et de labellisation, qu'ils soient internationaux, nationaux ou régionaux, peuvent offrir un début de valorisation à différents degrés de ressources patrimoniales plus ou moins spécifiques de la région.

\section{Une région cumulant tous les indicateurs de la " périphérisation » (Kühn, Weck 2013)}

7 La région périphérisée (Kühn, Weck, 2013) se caractérise par un manque d'attractivité généralisé qui se traduit de plusieurs manières. Tout d'abord, le départ des populations du territoire, en particulier des jeunes et des actifs, conduit à un vieillissement important et à une fragilisation économique généralisée. Cette situation démographique et économique peut entraîner une stigmatisation comme région en crise ou qui se vide, sensible dans le discours des acteurs locaux, mais également dans les médias, régionaux ou supra-régionaux. La région de Demmin, au centre du Land de Mecklembourg-Poméranie, apparaît donc comme une région périphérisée : elle a perdu plus d'un cinquième de sa population entre 1991 et 2011, principalement au cours de vagues de départs à la Réunification (Werz, Nuthmann, 2004), est particulièrement vieillissante et a une réputation de région en décroissance, fréquemment réactualisée dans la presse (Neu, 2006): «Rostock grossit, Demmin rétrécit»4. Cette image préoccupe, comme on peut le lire dans la stratégie de développement de la région LEADER Demminer Land, dans la catégorie risques du diagnostic territorial de la région: "Image déclinante de région affaiblie structurellement ${ }^{5}$ ». Cette même stratégie se donne pour objectif de "restaurer l'estime de soi de la région». Le manager régional dit lui-même ne pas se sentir en concurrence avec les autres régions LEADER du Land étant donné la situation de la région dont il a la charge : 
«Je me suis affranchi de tout ça, car pourquoi devrais-je être en concurrence avec eux ? Je sais que je suis dans une région rurale particulièrement difficile. » Manager de la région LEADER Demminer Land, 28.03.19 (trad. personnelle) relationnelle de la décroissance, et met en avant les relations de dépendance politique et économique des régions périphérisées. La région de Demmin est ainsi éloignée des grands centres de décisions politiques, comme Schwerin, où se situe le parlement du Land, mais également de Neubrandenburg, la préfecture du district depuis la grande réforme territoriale du Land de 2011. Demmin était auparavant la préfecture du district de Demmin (Demminer Land), qui a fusionné avec deux autres districts pour former le très grand district du Plateau des lacs mecklembourgeois. Cet éloignement est fréquemment évoqué comme une difficulté supplémentaire pour le Land :

« Nous avons naturellement l'inconvénient d'être en bordure du plus gros district du Mecklembourg-Poméranie, et même de toute la République fédérale. Nous sommes en bordure, au nord du district du Plateau des lacs mecklembourgeois. (...) C'est sûr, nous avons considérablement perdu du transfert [de l'administration du district] à Neubrandenburg. "

Maire de Demmin, 14.06.18 (trad. personnelle)

Le territoire dépend économiquement des subventions de l'État, mais il subit également une dépendance économique via les quelques grandes entreprises présentes sur son territoire ; il dépend par exemple de la fermeture de filiales décidée depuis le siège des grandes entreprises, situées hors du Land. La petite ville de Dargun, proche de Demmin, tire son épingle du jeu grâce à une politique attractive pour ces filiales. La laiterie est ainsi l'un des plus gros employeurs de la région, mais la ville en reste dépendante.

«Tout le paysage de la laiterie s'est modifié. (...) En fait, toutes les petites entreprises ici et là ont été rachetées par les plus grandes. Et les plus grandes par des encore plus grandes. Et avec le temps, ils ne sont plus que quelques Konzern en Allemagne, on appartient à l'un de ceux-là. "

Maire de Dargun, 14.06.18 (trad. personnelle)

10 La situation de la région de Demmin présente donc peu d'opportunités de développement, de par le faible nombre d'entreprises de la région, le départ massif de ses forces vives et son image. Valoriser le patrimoine naturel et culturel de la région pourrait alors constituer une voie de développement possible.

\section{Quelle ressource patrimoniale dans les campagnes autour de Demmin?}

11 De manière générale, l'intervention d'acteurs extérieurs est souvent nécessaire à la reconnaissance du patrimoine (De Saint-Pierre, 2014). À l'échelle mondiale, l'UNESCO reconnaît des sites en tant que patrimoine mondial naturel ou culturel, leur apportant visibilité et reconnaissance internationale. Le patrimoine immatériel a également fait l'objet d'une reconnaissance, plus tardive, et a une importance particulière dans les espaces ruraux, car il permet de mettre en avant des traditions spécifiques à ces espaces. Cette reconnaissance mondiale de l'UNESCO ne concerne dans le Mecklembourg-Poméranie que les deux villes de Stralsund et Wismar, classées en 2002 en tant que villes de la hanse typiques ${ }^{6}$, même si le château de Schwerin, la capitale du Land, est également en cours de candidature, sous le nom «Kulturlandschaft de l'historisme romantique $»^{7}$. Le Land compte également trois réserves de biosphères reconnues par l'UNESCO, mais pas en son centre. La région de Demmin est donc à

Belgeo, 2 | 2021 
l'écart de cette reconnaissance. Enfin le patrimoine immatériel de cette région ne fait pas l'objet de reconnaissance particulière, même si une demande a été déposée pour certaines traditions du Land dans son ensemble (comme l'utilisation d'instruments de musique particuliers ${ }^{8}$ ).

À l'échelle fédérale, le Land comporte trois des seize Parcs Nationaux allemands : les paysages du lac de Müritz, du lagon de Poméranie-occidentale et de la presqu'île de Jasmund sont ainsi reconnus comme exceptionnels et à préserver, mais ne concernent pas la région de Demmin. Si l'ancienne frontière interallemande a fait l'objet de projets de patrimonialisation dans d'autres Länder de l'Est (Quéva, 2012b), ce n'est pas le cas dans le Mecklembourg-Poméranie. En revanche, la forêt de chênes millénaires d'Ivenack, dans les environs de Demmin, constitue le seul site classé à rayonnement national de la région. Il s'agit du premier monument national naturel reconnu comme tel en 2016. Cette réserve est donc considérée comme un héritage à préserver, constitutif de l'identité nationale, au cœur de la zone d'étude.

Figure 2. Carte du patrimoine classé du Mecklembourg-Poméranie.

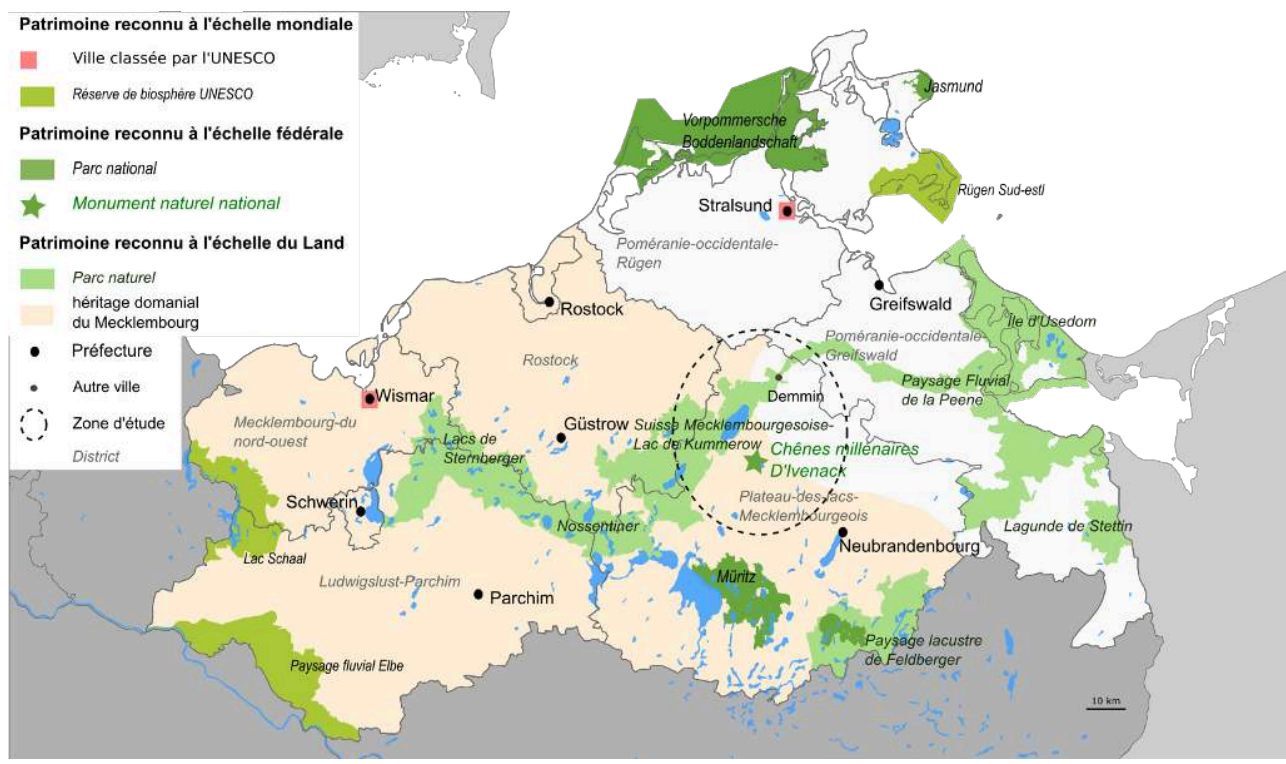

J. Lécuyer, 2020

13 À l'échelle du Land, la région de Demmin comporte deux des sept parcs naturels, le parc «Suisse mecklembourgeoise - lac de Kummerow» et une partie du parc «Paysage fluvial de la Peene», qui valorisent les paysages de la région. Plus localement et récemment, quelques responsables touristiques de la région ont tenté de valoriser le marais autour de Demmin (une grande tourbière), dont la spécificité et la surface sont remarquables.

«Et en tant que responsable du tourisme, on a beaucoup travaillé à valoriser les marais. (...) C'est incroyable. Tout est grand, on voit ces étendues d'eaux, des îles, et tout plein d'oiseaux, cela attire des ornithologues, des photographes, c'est vraiment un petit lieu très particulier dans lequel on pourrait faire beaucoup plus. »

Coordinatrice du tourisme du Lac de Kummerow, 3.05.19 (trad. personnelle)

14 Cette démarche en est cependant encore à ses débuts, et certains acteurs de la région eux-mêmes n'en ont pas connaissance. En revanche, la valorisation du patrimoine bâti est, elle, plus ancienne, et beaucoup de bâtiments sont protégés en tant que 
monuments ("Denkmalschutz»). Les plus caractéristiques dans la région sont les manoirs et petits châteaux, très nombreux dans la région, témoins de l'héritage domanial du Mecklembourg (Halama, 2012). Si certains ont été restaurés ou réhabilités, beaucoup d'entre eux sont laissés à l'abandon faute de moyens, la restauration d'un bâtiment classé devant se faire selon des normes spécifiques souvent coûteuses. Le patrimoine de la région à valoriser est donc plutôt naturel, ce qui correspond à une tradition ancienne en Allemagne.

\section{La ressource paysagère, une valorisation ancienne en Allemagne}

En Allemagne, la protection du patrimoine a depuis longtemps surtout porté sur le paysage et la nature (von Hirschhausen, Lacquement, 2007). En effet, comme le montre E. Vonau, la construction du paysage en ressource est très liée à l'émergence des mouvements de protection de la nature du XIX ${ }^{\mathrm{e}}$ siècle, aussi bien en France qu'en Allemagne ; la nature devient une ressource à protéger, en parallèle de la constitution d'un patrimoine culturel national avant tout fondé sur les œuvres d'arts puis les monuments. La protection devient le reflet d'une vision patrimoniale de la nature, alors perçue comme paysagère, et lui donne une forte valeur culturelle et symbolique dans la construction de l'histoire nationale. Protéger la nature revient donc à protéger une ressource nationale, ce qui justifie l'intervention des pouvoirs publics (Vonau, 2012).

Dans le cas allemand, la notion de Kulturlandschaft est centrale dans la mise en valeur $\mathrm{du}$ patrimoine, en particulier paysager (Lacquement, 2012 ; Vonau, 2012). Cette notion difficilement traduisible, littéralement « paysage cultivé ou culturel » étant donné le double sens de Kultur en allemand, peut faire référence à la fois au paysage agraire, mais également au patrimoine paysager, au paysage hérité. En insistant sur la dimension anthropisée du paysage, la Kulturlandschaft s'oppose à la nature sauvage (Wildernis), et c'est ce terme, dans ses différentes significations, que l'on retrouve pour parler de patrimoine paysager dans les politiques d'aménagement allemandes. Forte de cette ressource paysagère à la valorisation ancienne en Allemagne, la région de Demmin pourrait saisir cette opportunité pour se développer. Le contexte de périphérisation vient néanmoins limiter ses perspectives de développement.

\section{Le développement rural par la valorisation du patrimoine, limité par le contexte de périphérisation}

17 Les tentatives de développement rural par la valorisation du patrimoine sont particulièrement encouragées par les pouvoirs publics car ils leur prêtent toutes les vertus : concilier développement durable, aménagement global et intégré du rural, et prise en compte de la diversité des identités régionales (Schenk, 2012). En Allemagne, la mise en patrimoine des espaces ruraux s'est faite en deux temps.

\section{La mise en patrimoine des campagnes allemandes}

18 Le changement fonctionnel des campagnes qui s'est opéré à l'Ouest dans les années 1980 se fait principalement par une diversification des activités dont la mise en patrimoine est le support, et il s'opère de même à l'Est à la Réunification : en cherchant de nouveaux potentiels de développement, les acteurs locaux redécouvrent les 
attributs physiques des lieux, leur héritage culturel et historique, ce qui les conduit à " réinventer » un patrimoine rural (von Hirschhausen, Lacquement, 2007). C'est ce patrimoine que les politiques publiques encouragent à utiliser comme base du développement rural, officiellement avec la Loi d'aménagement du territoire de 1998 (Raumordnungsgesetz). L'État fédéral poursuit dans la lignée de cette loi en inscrivant en 2006 la valorisation des paysages anthropisés (Kulturlandschaften) comme l'un des trois principaux schémas d'orientation de la politique d'aménagement du territoire allemand (Schenk, 2012).

19 Ces cadres législatifs sollicitent donc toujours plus les acteurs locaux, qu'ils encouragent à construire eux-mêmes leurs projets de développement, rapprochant ainsi développement rural et développement local. Si cette mise en patrimoine doit se faire "par le bas", avec un inventaire par les acteurs locaux de leurs ressources patrimoniales, toute latitude n'est pas laissée à ces derniers: dans un souci de cohérence, des experts sont mandatés par les échelons politiques supérieurs pour dresser la liste de tout ce qui constitue une ressource potentielle à conserver ou valoriser, en raison de son intérêt esthétique, écologique ou identitaire (von Hirschhausen, Lacquement, 2007). L'identification se fait donc à l'échelon local, mais sous l'impulsion et selon les directives d'acteurs extérieurs au territoire.

\section{L'acquisition progressive d'un savoir-faire}

L'acquisition du savoir-faire que représente cette mise en patrimoine « par le bas » est plus difficile dans les Länder de l'Est. Cette démarche y est moins évidente, encore trente ans après la Réunification, et avoir appartenu à la RDA est un facteur d'inertie qui reste à dépasser, en particulier pour les plus âgés (Quéva 2012a). Comme le soulignent B. von Hirschhausen et G. Lacquement, la construction du patrimoine rural révèle la dichotomie des formes de structuration territoriale à l'Est et à l'Ouest (von Hirschhausen, Lacquement, 2007). Les espaces ruraux orientaux ont en effet dû faire face à plusieurs grands bouleversements à la réunification. La décollectivisation, menée très rapidement, a conduit à la marginalisation de l'activité agricole dans les sociétés locales, favorisant la diversification fonctionnelle des espaces ruraux, et donc la prise en compte potentielle de nouvelles ressources rurales autres que l'agriculture. En parallèle, l'inversion des politiques publiques, c'est-à-dire le passage d'une gestion planifiée au sein de régions de planification à un développement endogène s'appuyant sur des régions de projets, a représenté un changement supplémentaire (Lacquement, 2010). Les changements post-socialistes affectent donc la trajectoire de ces espaces, trajectoires qui dépendent du contexte géographique de réception de ces changements, mais également du passé de ces territoires (ibid). Dans le cas de la mise en patrimoine du Mecklembourg, l'héritage domanial est à prendre en compte puisqu'il constitue une grande partie des ressources à valoriser. Cependant, l'héritage post-socialiste de planification " par le haut » joue également un rôle, il freine le développement local en raison du manque de savoir-faire des acteurs locaux.

De fait, le processus de mise en patrimoine s'effectue en plusieurs temps : inventaire, caractérisation, préservation/restauration, promotion (Lacquement, 2012). Or, l'identification de ce qui peut constituer un projet de valorisation viable ne va pas de soi. Comme l'indique le manager de la région LEADER de Demmin, chacun est persuadé d'avoir un projet unique, et est surpris d'apprendre que la même chose existe déjà 
quelques kilomètres plus loin'. Beaucoup de petits projets se montent donc plutôt individuellement, sans ancrage dans un réseau d'acteurs locaux. L'exemple type de la région est un projet de la réhabilitation d'un manoir ou d'un château.

Figure 3. Château de Kummerow (première restauration de 2012 à 2016 via LEADER).

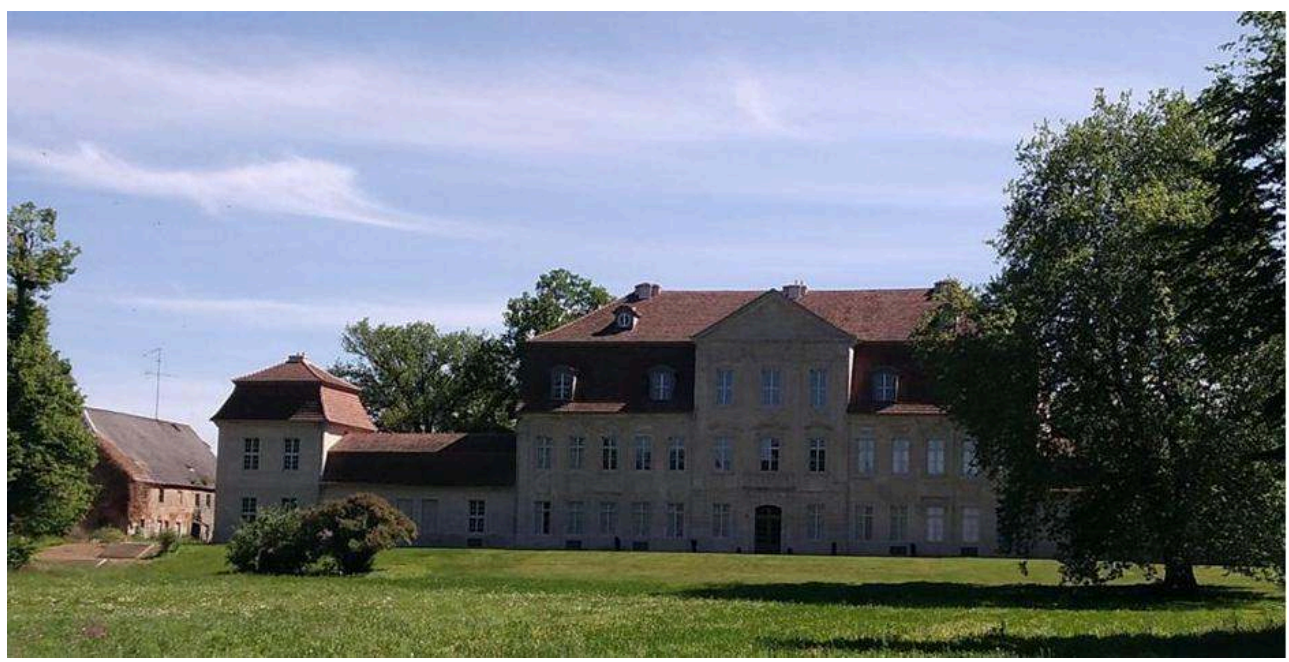

J. Lécuyer, 2018

Comme le montre A. Halama précisément au sujet de l'héritage domanial du Mecklembourg, très présent dans la région de Demmin :

«La remise en état et la valorisation économique de ce patrimoine nécessitent des investissements que les acteurs locaux sont loin de pouvoir avancer, ni sur leur fonds propres, ni en ayant recours aux dispositifs d'aides publiques régionales, nationales ou européennes. Il leur manque à la fois les ressources économiques, les réseaux sociaux et le savoir-faire. Ce sont donc surtout des acteurs extérieurs qui sont à l'origine de la plupart des projets. Ils apportent leurs moyens. "

(Halama, 2012)

Beaucoup des projets de la région de Demmin sont donc menés par des acteurs extérieurs au territoire (comme de nouveaux propriétaires habitant à Berlin), ayant la maitrise des procédures de projets sur lesquelles s'appuie désormais le développement rural y compris en Allemagne orientale (Lacquement, Quéva, 2016). Ce déficit d'apprentissage a cependant été identifié par les acteurs publics, et des réunions sont par exemple organisées par la préfecture du district, à Neubrandenburg; chacun peut y participer pour apprendre à monter un dossier (procédure à suivre, formulaires à remplir, documents à présenter, éléments de langage à faire figurer).

\section{Résistances et échecs du développement rural intégré}

La notion de développement rural intégré se développe dans les années 1990, sous la forme d'une nouvelle logique de gouvernance régionale en rupture avec l'aménagement "par le haut». Elle intègre aussi les principes du développement durable, ce qui conduit à adopter une approche intersectorielle des problématiques de développement, à valoriser les paysages ruraux régionaux, mais aussi à favoriser les démarches participatives (Grabski-Kieron, 2012). Le développement rural intégré suppose ainsi une démarche endogène portée par les acteurs locaux constitués en réseau, sur un territoire qui se superpose plus ou moins au maillage politique et 
administratif (Lacquement, 2012). Cette démarche demande donc l'implication des habitants; la question de l'intérêt des populations locales pour les ressources patrimoniales que l'on cherche à valoriser est alors centrale dans la réussite d'un projet. Dans notre région d'étude, l'ancienne manager régionale évoque ses difficultés à constituer un projet pour répondre à un appel du ministère fédéral de l'Agriculture et de l'Alimentation ${ }^{10}$, projet qui n'a finalement pas été retenu :

«Mais c'était tellement de travail... Mobiliser les gens ici... Les gens sont sceptiques par rapport aux nouvelles idées. (...) LEADER est un bon apprentissage de l'obtention de fonds, on connaît mieux les concepts-clés qui doivent impérativement figurer. Mais bon, c'est vrai que... Il faut aimer baratiner. (...) Mais c'est vraiment dur d'impliquer les gens du coin... »

Ancienne manager de la région LEADER Demminer Land, 22/05/2018 (trad. personnelle)

Cette résistance peut également se traduire par les difficultés rencontrées dans la pratique du développement rural intégré tel qu'il est prescrit par les politiques d'aménagement. La figure du manager, chargé de porter et de représenter le projet du territoire, ainsi que de coordonner les différents acteurs mobilisés, émerge sans que le reste des acteurs locaux ne maîtrise ces nouvelles méthodes apparentées au management. Malgré l'importance du manager dans la gestion des territoires ruraux allemands (Quéva, 2012a), ce sont souvent les réseaux préexistants qui fonctionnent le mieux, en Allemagne comme dans d'autres espaces ruraux soumis au même basculement vers une logique de développement par le projet (Quéva, 2007a; Bessière et al., 2016). Comme en témoigne le manager de la région LEADER Demminer Land, son travail n'est pour lui pas assez reconnu par le district, acteur traditionnel de l'aménagement du territoire, qui rechigne à les inclure, lui et ses collègues :

«Pour moi les grandes réunions du district sont comme une bourse aux contacts, où on a la chance de rencontrer des gens qui font aussi des choses bien, de mettre en place une coopération, de se mettre en réseau. Mais, je vais le formuler avec précaution... on ne nous invite pas toujours. »

Manager de la région LEADER Demminer Land, 23.05.18, (trad. personnelle)

Ce manque d'habitude à travailler en réseau et coordonner les différents acteurs d'un même territoire autour d'un projet est également sensible à Ivenack. La valorisation du parc de chênes millénaires, seule ressource territoriale classée de la région d'étude, et bénéficiant d'une grande attractivité, illustre bien le manque de coopération entre acteurs. 
Figure 4. Chênes millénaires d'Ivenack - nouveau chemin de promenade ajouté en 2018.

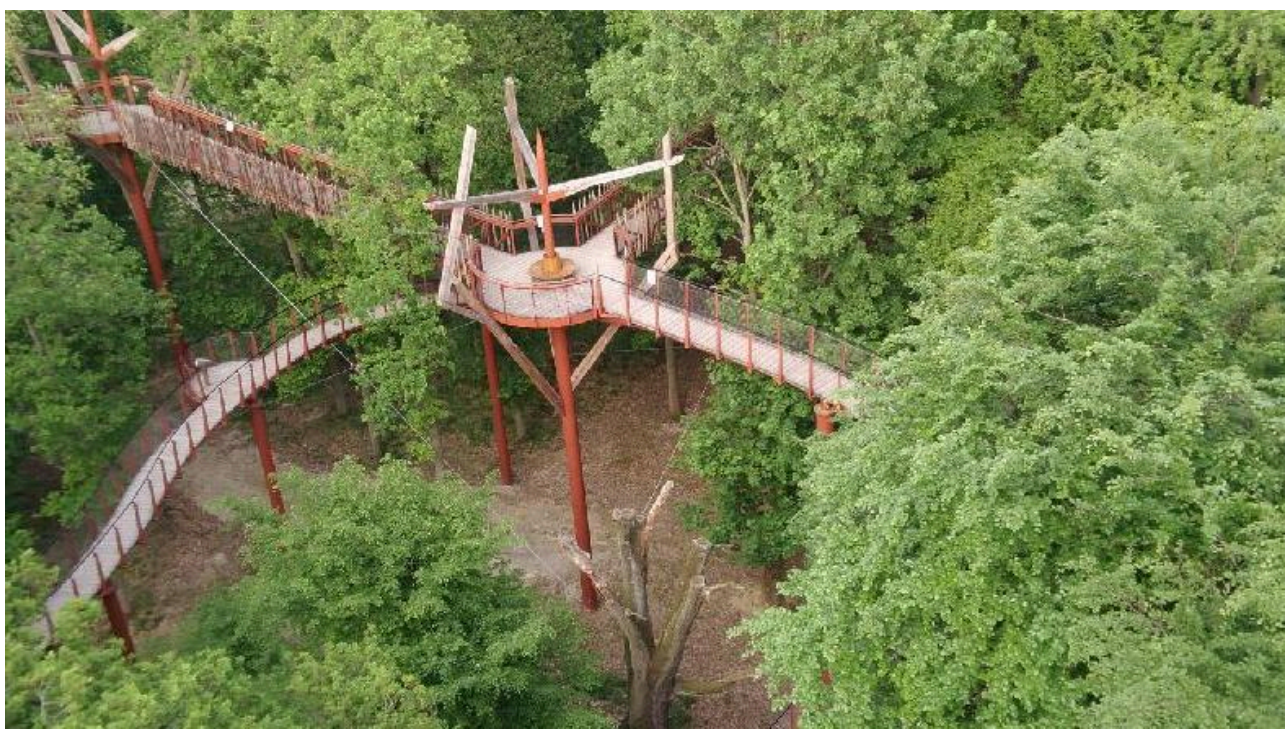

J. Lécuyer, mai 2018 boutique de souvenirs, stand de prospectus ou même sanitaires qui permettraient de retenir les visiteurs un peu plus longtemps dans la région. Ceux-ci poursuivent donc le plus souvent leur route jusqu'à la côte Baltique, plus attractive. Le projet de restauration du château voisin du parc d'Ivenack, en cours, se fait de la même manière sans aucune concertation avec les acteurs locaux, ce que déplore la coordinatrice touristique de la région dont le poste a été créé spécifiquement pour favoriser la communication et la création de réseaux : « tous agissent en parallèle, ils ne s'assoient jamais à la même table ${ }^{11}{ }^{\prime}$. Les procédures bottom-up, bien qu'au cœur du paradigme du développement rural intégré, sont donc maîtrisées très inégalement par les acteurs locaux, par manque d'intérêt ou de ressources financières ou en personnel.

\section{Projets de développement et patrimoine, une identité régionale renforcée ou menacée?}

«La référence au patrimoine est un levier de valorisation, de développement, de changement d'image et d'attractivité ; pour autant la question de savoir par qui et pour qui s'opère la patrimonialisation demeure importante » (Veschambre, 2015). Les enjeux de l'image sont particulièrement importants dans une région périphérisée, qui est souvent stigmatisée ou connotée négativement.

\section{Construire une identité régionale qui soit valorisable}

Les modes de valorisation du patrimoine rural s'appuient sur des stratégies qui sont les mêmes qu'ailleurs : produits locaux, marketing territorial, guides touristiques. D'un côté, on recherche l'authenticité, de l'autre, on banalise la ressource (Burggraaff, Kleefeld, 2012), augmentant le risque de construction d'une identité artificielle principalement à des fins de marketing. Dans les environs de Demmin, les acteurs locaux ont compris la nécessité de renforcer leur identité régionale pour pouvoir 
valoriser leur territoire, en changer l'image de manière à le rendre plus attractif. La constitution de cette identité commune ne va cependant pas de soi, et n'en est encore qu’à ses débuts.

\section{Encadré : construire une identité territoriale dans les campagnes autour de Demmin}

2014 : un couple engagé dans la vie de leur petit village cherche à promouvoir les produits locaux dans leur petit magasin, et décide pour cela de créer un réseau d'entrepreneurs locaux, sous le nom de Meck-Schweizer.

2017 : une coopérative et une association du même nom sont créées.

début 2019 : devant le succès de la marque, un groupe de divers acteurs locaux se forme pour tenter de formaliser une identité régionale à valoriser sous le nom de Suisse mecklembourgeoise. Quelques réunions préparatoires ont eu lieu en petit comité.

03.2019 : une réunion a lieu pour tenter d'établir un cahier des charges d'un label de produits locaux « Meck-Schweizer », en s'inspirant des chartes similaires d'autres régions.

05.2019 : une première réunion intitulée « Initiative pour renforcer l'identité régionale - la marque Suisse mecklembourgeoise » (Mecklenburgische Schweiz) a lieu pour débattre de ces questions en impliquant davantage d'acteurs. Ont notamment été conviés des représentants du district du Plateau des Lacs mecklembourgeois (dont fait partie la région de Demmin) et du district voisin, des élus locaux, une pasteure, des représentants du tourisme et du parc naturel « Suisse mecklembourgeoise », mais aussi bien sûr les membres fondateurs du réseau Meck-Schweizer, déjà bien établi. La presse avait également été invitée, et des articles paraissent régulièrement dans les quotidiens locaux pour relayer les activités du groupe ${ }^{12}$. D'après une des fondatrices de l'initiative, beaucoup de ces acteurs récemment impliqués ne reviendront cependant pas aux réunions suivantes, en particulier les acteurs institutionnels qui travaillent à l'échelon des districts : il s'agit avant tout de fédérer des acteurs à un échelon très local, d'autres personnes plus impliquées localement viendront donc les remplacer.

G. Di Méo pointe justement une gémellité entre territorialité et patrimonialité, en ce que toutes deux sont fondatrices de lieux signifiés, en particulier lorsqu'il est question d'assoir et de valider des procédures de développement local, ou qualifier ses produits (Di Méo, 2007). Il divise le processus de territorialisation en plusieurs étapes, parfois concomitantes: délimitation, singularisation, exposition (choix $\mathrm{du}$ nom et représentation), valorisation, puis " adoption ", c'est-à-dire appropriation. Selon cette grille de lecture, la campagne autour de Demmin en serait au cœur de l'étape de délimitation/singularisation. Au cours de la réunion mentionnée ci-dessus, la nécessité de critères pour définir la Suisse mecklembourgeoise est évoquée à plusieurs reprises. Ce terme est déjà employé (une des éditions locales du quotidien Nordkurier porte par exemple ce nom), mais sans renvoyer à un territoire précis. Les personnes présentes décident de se fédérer autour d'un paysage typique, mais peinent à le définir 
nettement: collines, lacs, qu'est ce qui est vraiment spécifique? Se distinguer de régions proches aux ressources patrimoniales souvent similaires est difficile.

Le gérant d'un hôtel conforte cette idée: peu de gens connaissaient la Suisse mecklembourgeoise. Il interroge les autres sur ce qui fait la Kulturlandschaft, et demande s'il y en a vraiment une spécifique à la région. Aucune réponse ne fait l'unanimité.

Compte rendu d'observation, réunion du 6.05.2019, Karnitz

La question des limites physiques de la région est également longuement débattue : qui fait partie de la Suisse mecklembourgeoise, qui en est exclu? Les avis sont loin d'être unanimes. À des critères paysagers comme les collines, les champs ou les lacs, se mêlent des critères économiques : faut-il dissocier la zone de Kummerow, qui essaie elle aussi de se faire un nom en tant que lieu touristique d'importance comportant un grand lac, un château, un lieu d'exposition de photographes renommés? La question reste sans réponse claire, mais la présence de la coordinatrice touristique de Kummerow semble indiquer que non.

De plus, une sélection s'opère dans la mise en récit du patrimoine à valoriser. En tant que région périphérisée, les campagnes de Demmin sont déjà associées au vide et au déclin. Il s'agit avant tout pour les acteurs locaux de changer cette image pour être plus attractifs. Cette mise en récit du patrimoine régional doit donc se faire de manière simple et attrayante, ce qui est souvent difficile étant donné l'histoire de la région et les images qui peuvent y être encore associées. Le nom de la ville de Demmin est par exemple associé à une vague de suicide en 1945 et à des marches néonazies ayant lieu les 8 mai (Iost, 2012). La recherche d'authenticité, de parcours de vie typiques s'avère parfois délicate :

Une responsable des programmes de développement rural du Land explique que ce qui fait la spécificité de la Suisse mecklembourgeoise, ce sont les gens, ce sont les histoires, les récits de vie, plus que la définition géographique. (...) La pasteure prend la parole, et rappelle que les histoires de vie que l'on veut mettre en valeur sont son quotidien, que ce ne sont pas de belles histoires à valoriser mais des histoires de violence et de fuite qui ne font ni une belle identité ni une bonne publicité.

Compte rendu d'observation, réunion du 6.05.2019, Karnitz

Une forme d'urgence à se fédérer autour d'un nom, d'une identité, se fait sentir : « Il faut croire en notre identité sinon personne n'y croira ", répond un acteur très engagé dans la promotion de la région à un vieil homme affirmant que peu de gens se revendiquent de la Suisse mecklembourgeoise. Selon la typologie des récits territoriaux proposée par certains auteurs en fonction du rapport au temps des récits territoriaux (Debarbieux et al., 2001), la Suisse mecklembourgeoise a une position ambiguë. L'histoire de cette campagne et de ses habitants est fréquemment évoquée, mais il ne s'agit pas tant de valoriser un passé glorieux que de ne pas évoquer un passé stigmatisant. À plusieurs reprises, la dimension marketing de l'identité que l'on cherche à formaliser est évoquée :

Un vieil homme rappelle que l'ancien district de Demmin a essayé de se valoriser en tant que "Demminer Land» [Campagne de Demmin] mais que cela n'a pas marché.

(...) Selon lui, il faut faire attention à ne pas faire une marque de choses qui ne se vendent pas du tout.

Compte rendu d'observation, réunion du 6.05.2019, Karnitz Poméranie-occidentale en général. Selon la coordinatrice du lac de Kummerow, la ville 
de Demmin refuse pour cette raison de valoriser son patrimoine lié à son appartenance à la Poméranie (principalement des bâtiments clés et le rôle de la ville à cette époque), par peur de renforcer le stigmate. C'est aussi pour cette raison que les campagnes autour de Demmin se tournent plutôt vers l'Ouest, et cherchent à être associées aux campagnes mecklembourgeoises.

\section{Des projets de valorisation patrimoniale renforçant la situation de dépendance de la région}

Comme vu plus haut, la valorisation du patrimoine est souvent promue dans les politiques publiques comme vecteur de développement des territoires ruraux. Pourtant, dans la région de Demmin, cette mise en patrimoine semble au contraire renforcer la situation de dépendance, caractéristique des régions périphérisées. Tout d'abord, l'impulsion de cette mise en patrimoine vient des échelons supérieurs, comme par exemple la loi fédérale valorisant la Kulturlandschaft. Ensuite, le rayonnement des sites classés vient en grande partie de la certification dont ils bénéficient, et les labels les plus prestigieux sont également décidés en dehors de la région, par exemple pour la labellisation nationale comme les monuments naturels nationaux. Par ailleurs, S. Depraz a montré dans le cas du parc national de Müritz que ces espaces classés et protégés deviennent avec l'obtention d'un label le "patrimoine d'une nation ", et risquent finalement d'être dépossédés de leur caractère d'espace de vie local. L'instauration d'un espace protégé peut ainsi être vécue comme une dépossession symbolique : ce qui était territoire de vie, repère identitaire voire lieu de mémoire pour les habitants se trouve frappé par de nombreux interdits (Depraz, 2007). La mise en patrimoine peut donc comporter un risque supplémentaire de perte de pouvoir des acteurs locaux sur leur territoire. Enfin, d'un point de vue financier, les projets de développement s'appuyant sur le patrimoine régional, comme la mise en valeur de paysages typiques par des circuits touristiques ou la réhabilitation d'un manoir, se font avec des fonds extérieurs. Il s'agit par exemple de fonds européens (LEADER notamment), ou de fonds privés de personnes extérieures à la région ayant acheté une propriété.

Mais surtout, cette mise en patrimoine s'effectue principalement à destination d'une clientèle extérieure à la région, le plus souvent même extérieure au Land. Cette région déjà sous influence met donc en valeur ses paysages, son bâti classé, de manière à les rendre attractifs à d'autres, souvent citadins, pour qu'ils correspondent à leurs goûts et leurs attentes (Lacquement, 2012). C'est par exemple le cas des descendants des anciennes familles domaniales qui, revenant sur les lieux à la Réunification, décident de racheter leurs domaines familiaux :

«Ils savent aussi mobiliser le passé en termes de marketing pour promouvoir leurs produits touristiques auprès d'une clientèle berlinoise ou hambourgeoise choisie » (Halama, 2012).

Dans ce cas, la mise en patrimoine demandée par les politiques publiques se fait donc sur injonction d'échelons politiques supérieurs, par des personnes qui n'habitent pas forcément le territoire, pour une population extérieure au territoire. 


\section{Conclusion}

Dans une région rurale périphérisée comme la région de Demmin, les difficultés démographiques, socio-économiques et politiques qui s'accumulent font du patrimoine une des rares ressources territoriales valorisable pour le développement, notamment via des labels et certifications. Cette mise en patrimoine est encouragée par les pouvoirs publics, pour qui la valorisation permet l'apprentissage des principes du développement rural intégré, particulièrement important dans les campagnes estallemandes marquées par l'héritage socialiste de planification. Cet apprentissage ne se fait pas sans heurts, et peut freiner certains projets de développement. En outre, la mise en patrimoine censée renforcer l'identité régionale peut parfois au contraire inciter à la création d'identité quelque peu artificielle dans un objectif de valorisation touristique.

La valorisation du patrimoine, naturel ou culturel, de cette région périphérisée pourrait donc finalement contribuer à renforcer sa situation de dépendance. L'impulsion de cette mise en patrimoine se situe souvent en dehors du territoire, issue d'une volonté politique supérieure ; les certifications les plus valorisées sont également décidées à l'échelon international ou national. De plus, les gros projets de restauration du patrimoine bâti sont souvent menés par les propriétaires des manoirs et châteaux, principalement citadins et ne vivant pas sur place, sans que la population locale soit toujours concertée. Enfin, cette mise en valeur du patrimoine se fait principalement à destination d'un public citadin, et les infrastructures créées le sont souvent selon les goûts de cette clientèle espérée.

\section{BIBLIOGRAPHIE}

BESSIÈRE J., CLARIMONT S. \& VLES V. (2016), « La ressource patrimoniale, outil de diversification touristique ? Le pôle d'excellence rurale du Néouvielle, entre innovation et recyclage ", Natures Sciences Sociétés, 24, 3, pp. 217-229.

BURGGRAAFF P., KLEEFELD K. (2012), « La conversion de l'économie régionale dans l'Eifel : de la "Sibérie rhénane" au parc de loisirs », Réinventer les campagnes en Allemagne, Sociétés, espaces, temps, Lyon, ENS Editions, pp. 91-103.

COPUS A., NOGUERRA J. (2016), “3. Conceptual and methodological Framework”, Inner peripheries: national territories facing challenges of access to basic services of general interest, ESPON 2020, pp. 22-33.

DEBARBIEUX B., FOURNY M.-C. \& VANIER M. (2001), « La prospective est représentation », Territoires 2020, 3, pp. 27-43.

DEPRAZ S. (2007), «Campagnes et naturalité : la redéfinition d'un rapport à la nature dans les espaces ruraux des nouveaux Länder », Revue d'études comparatives Est-Ouest, 38, 3, pp. 135-142. 
DE SAINT-PIERRE C. (éd.) (2014), La Ville patrimoine : formes, logiques, enjeux et stratégies, Rennes, Presses universitaires de Rennes.

DI MÉO G. (2007), « Processus de patrimonialisation et construction des territoires », Colloque « Patrimoine et industrie en Poitou-Charentes : connaître pour valoriser », Septembre 2007, PoitiersChâtellerault, France, pp. 87-109.

FLORENTIN D., FOL S. \& ROTH H. (2009), « La "Stadtschrumpfung” ou "rétrécissement urbain" en Allemagne : un champ de recherche émergent ", Cybergeo, $19 \mathrm{p}$.

GRABSKI-KIERON U. (2012), « Le développement rural intégré en Allemagne : une démarche de planification et d'action prise entre ambitions et réalités ", Réinventer les campagnes en Allemagne, Sociétés, espaces, temps, Lyon, ENS Editions, pp. 185-198.

HALAMA A. (2012), « Le passé au secours de l'avenir ? L'héritage domanial du Mecklembourg et sa valorisation contemporaine ", Réinventer les campagnes en Allemagne, Sociétés, espaces, temps, Lyon, ENS Editions, pp. 137-152.

IOST D. (2012), Géopolitique de l'extrême droite dans les nouveaux Länder, Thèse de doctorat, Université de Paris VIII.

KEIM K.-D. (2006), « Peripherisierung ländlicher Räume - Essay », APUZ, 37, pp. 3-7.

KÜHN M., WECK S. (2013), « Peripherisierung - ein Erklärungsansatz zur Entstehung von Peripherien », in BERNT M., LIEBMANN H. (dir.), Peripherisierung, Stigmatisierung, Abhängigkeit ? Deutsche Mittelstädte und ihr Umgang mit Peripherisierungsprozessen, Wiesbaden, Springer VS., p. 24-46.

LACQUEMENT G. (2010), Lire et analyser la transformation post-socialiste dans les territoires ruraux d'Allemagne orientale, Mémoire d'habilitation à diriger des recherches, Université Lumière Lyon 2, $184 \mathrm{p}$.

LACQUEMENT G., QUÉVA C. (2016), « Introduction - Innovations sociales et développement des territoires dans les campagnes européennes », Norois, 4, 241.

LACQUEMENT G., VON HIRSCHHAUSEN B. (2012), in BORN K.M. (éd.), Réinventer les campagnes en Allemagne : paysages, patrimoine et développement rural, Lyon, ENS Editions.

NEU C. (2006), « Territoriale Ungleichheit - eine Erkundung », Aus Politik und Zeitgeschichte, 37, pp. 8-15.

QUÉVA C. (2007a) « Acteurs et territoires du développement rural en Allemagne orientale. L'exemple de projets de développement régional dans la Thuringe du Sud-Ouest », Revue d'études comparatives Est-Ouest, 38, 3, pp. 117-134.

QUÉVA C. (2007b), « Les paradoxes de la Région en Allemagne, entre réseaux et territoires : la région, outil de déterritorialisation?", Annales de géographie, 653, pp. 41-64.

QUÉVA C. (2012a), « "Marketing régional” et valorisation du rural en Allemagne : discours et argumentaires des acteurs locaux dans la construction de nouveaux territoires ", Réinventer les campagnes en Allemagne, Sociétés, espaces, temps, Lyon, ENS Editions, pp. 199-212.

QUÉVA C. (2012b), « Entre intégration et patrimonialisation (trans-)frontalières : les dynamiques territoriales de l'ancienne frontière interallemande ", Cahiers de Géographie du Québec, 56, 159, pp. 525-541.

ROTH H. (2016), « Du déclin à la périphérisation : quand les courants constructivistes et critiques revisitent les différenciations spatiales en Allemagne », Cybergeo, https:// journals.openedition.org/cybergeo/27389. 
SCHENK W. (2012), « De l'espace résiduel aux paysages culturels : les nouvelles conceptions de la ruralité et de ses ressources dans les politiques territoriales en Allemagne », Réinventer les campagnes en Allemagne, Sociétés, espaces, temps, Lyon, ENS Editions, pp. 43-58.

VESCHAMBRE V. (2015), « Inventions patrimoniales et enjeux sociaux », Perspective, 1, pp. 191-198.

VON HIRSCHHAUSEN B., LACQUEMENT G. (2007), « Modèles de l'Ouest, territoires de l'Est. Le développement local et la valorisation du patrimoine dans les campagnes d'Allemagne orientale ", Revue d'études comparatives Est-Ouest, 38, 3, pp. 5-29.

VONAU E. (2012), « Le paysage et ses lectures : le mouvement de protection de la nature en France et en Allemagne au début du siècle », Réinventer les campagnes en Allemagne, Sociétés, espaces, temps, Lyon, ENS Editions, pp. 29-42.

WERZ N., NUTHMANN R. (Hrsg.) (2004), Abwanderung und Migration in Mecklenburg und Vorpommern, Wiesbaden, VS Verlag für Sozialwissenschaften.

\section{NOTES}

1. Abrégé par la suite en Mecklembourg-Poméranie.

2. On entend ici la région au sens large (Region), qui désigne un espace d'action local fondé sur des coopérations souvent réticulaires et informelles (Quéva, 2007b), contrairement à la région d'aménagement ou de planification.

3. Programme de développement rural européen (Liaison Entre Actions de Développement de l'Économie Rurale) fondé sur des appels à projets. Les acteurs locaux doivent se constituer en « région LEADER » pour bénéficier des fonds.

4. "Rostock wächst, Demmin schrumpft», 20.08.2019 14:34 Uhr, NDR 1 Radio MecklenburgVorpommern, https://www.ndr.de/nachrichten/mecklenburg-vorpommern/ Bevoelkerungsprognose-MV-schrumpft-langsamer,bevoelkerung152.html (consulté en avril 2020).

5. "Sinkendes Image einer strukturschwachen Region" (Stratégie de développement de la région LEADER Demminer Land pour la période 2014-2020, p. 52).

6. https://www.regierung-mv.de/Landesregierung/bm/Kultur/Sparten/Welterbe/ (consulté en avril 2020).

7. https://www.regierung-mv.de/Landesregierung/bm/Kultur/Sparten/Welterbe/ Weltkulturerbe\%E2\%80\%93Bewerber-in-MV/ (consulté en avril 2020), https://www.welterbebewerbung-schwerin.de/ (consulté en avril 2020). https://www.regierung-mv.de/Landesregierung/bm/Kultur/Sparten/Welterbe/ Immaterielles-Kulturerbe/ (consulté en avril 2020).

9. Entretien manager de la région LEADER Demminer Land, 28.03.19.

10. Le ministère fédéral de l'Agriculture et de l'Alimentation (BMEL) a lancé en 2017 une grande initiative fédérale pour le développement rural, constituées de plusieurs programmes de développement qui fonctionnent par appel à projet.

11. Coordinatrice du Lac de Kummerow, 3.05 .19 (trad. personnelle).

12. 10.06.2019, « Die Mecklenburgische Schweiz will selbstbewusster werden », Nordkurier, p. 14. 


\section{RÉSUMÉS}

La région rurale de Demmin, située dans le nord-est de l'Allemagne, est touchée par la périphérisation, c'est-à-dire qu'elle cumule départs de population, affaiblissement économique, dépendance politique et stigmatisation. La valorisation du patrimoine naturel et culturel apparaît alors comme l'un des rares moyens de développer la région. Le présent article interroge dans quelle mesure cette mise en patrimoine contribue au développement local ou renforce la situation de dépendance de la région. Les projets de valorisation du patrimoine rural sont souvent encouragés par les politiques publiques, car ils permettent aux acteurs locaux de se fédérer autour d'un projet qu'ils conçoivent eux-mêmes à partir des ressources de leur territoire. Cependant, le contexte post-socialiste et de périphérisation de la région limitent les bienfaits supposés de ce mode de valorisation. En outre, le renforcement de l'identité régionale et la mise en valeur du patrimoine se font principalement à des fins de marketing, et à destination d'une clientèle extérieure au territoire. Le risque de perte de pouvoir des acteurs locaux sur leur territoire en est donc accru.

The rural region of Demmin, located in the north-east of Germany, is affected by peripheralization: population departures, economic weakening, political dependence and stigmatization. The promotion of the natural and cultural heritage could therefore be a way of developing the region. The article questions the extent to which the enhancement of heritage contributes to local development or reinforces the situation of dependency of the region. Heritage-based development projects are often encouraged by public policies, as they allow local actors to federate around a project that they design themselves from the resources of their own territory. However, the post-socialist and peripheralization context of the region limits the supposed benefits of this mode of valorization. Moreover, the strengthening of regional identity and the enhancement of heritage are mainly for marketing purposes, and for customers outside the territory. The risk of local actors losing power in their territory is therefore increased.

\section{INDEX}

Mots-clés : patrimoine rural, développement local, développement rural, périphérisation, Allemagne, nouveaux Länder, Mecklembourg-Poméranie-occidentale

Keywords : rural heritage, local development, rural development, peripheralization, Germany, new Lander, Mecklenburg-West Pomerani

\section{AUTEUR}

\section{JOSÉPHINE LÉCUYER}

Doctorante, Université Paris 1 Panthéon-Sorbonne, UMR 8504 Géographie-cités, équipe P.A.R.I.S, Josephine.Lecuyer@univ-paris1.fr 